TITLE:

\title{
Measuring deformations using SAR interferometry and GPS observables with geodetic accuracy: Application to Tokyo, Japan
}

\author{
$\operatorname{AUTHOR(S):~}$ \\ ElGharbawi, Tamer; Tamura, Masayuki
}

\section{CITATION:}

ElGharbawi, Tamer ...[et al]. Measuring deformations using SAR interferometry and GPS observables with geodetic accuracy: Application to Tokyo, Japan. ISPRS Journal of Photogrammetry and Remote Sensing 2014, 88: 156-165

\section{ISSUE DATE:}

2014-02

URL:

http://hdl.handle.net/2433/182053

\section{RIGHT:}

(C) 2013 International Society for Photogrammetry and Remote Sensing, Inc. (ISPRS). Published by Elsevier B.V.; この論文は出版社版でありません。引用の際には出版社版を ご確認ご利用ください。; This is not the published version. Please cite only the published version. 


\title{
Measuring Deformations using SAR Interferometry and GPS Observables with Geodetic Accuracy: Application to Tokyo, Japan
}

\begin{abstract}
This paper presents new methodology for correcting interferometric synthetic aperture radar (InSAR) deformation maps using GPS observables and products. The methodology presents a sequential procedure for correcting the errors presented in InSAR deformation maps such as troposphere delay, ionosphere delay and baseline error. The main target of this research is to measure land deformations with geodetic accuracy using only one Lband interferogram with the aid of GPS observables and products. The proposed methodology was tested on Tokyo bay area which has been affected by the 2011 Tohoku earthquake. The results were verified against deformations detected by GPS stations and geodetic triangulation network showing a standard deviation of 5.6 and 10.5 millimeters, respectively.
\end{abstract}

\section{Keywords}

InSAR; GPS; GSI; 2011 Tohoku Earthquake; Land Deformation Measurement.

\section{Introduction:}

Interferometric synthetic aperture radar (InSAR) has been used successfully to measure and study surface deformation due to several phenomena such as glacier movements (Goldstein et al., 1993), earthquakes (Massonnet et al., 1994) and land subsidence (Buckley et al., 2003). However, InSAR presents some limitations. For example; it is highly affected by atmospheric variations such as tropospheric and ionospheric delay; the quality of InSAR measurements is also affected by the quality of the satellite orbital data which can be identified as additional long wave interferometric fringes. Moreover deformation values obtained from InSAR is subjected to phase unwrapping errors and presents relative deformation values not absolute.

The integration between InSAR and GPS is not new; many researches have presented and established the improvements which can be obtained by using the GPS observables in correcting the interferometric deformation maps. For correcting tropospheric delay artifact presented in InSAR (Li et al., 2006) and (Song et al., 2008) have incorporating GPS derived Zenithal Tropospheric Delay (ZTD) with a topography-dependent turbulence model based on Kolmogorv turbulence theory, (Onn and Zebker, 2006) have used an exponential altitudedependent model derived from the GPS ZTD and presented the incorporation of Taylor's "frozen-flow" hypothesis, (Li et al., 2004) have developed an elevation-dependent model based on the integrated use of GPS and ground meteorological observations, (Xu et al., 2006) and (Janssen et al., 2004) have used the ZTD derived from continuous GPS network and kriging algorithm to correct for the tropospheric delay at Tokyo area, Japan.

For correcting baseline errors (Zhang et al., 2008) proposed a bilinear algorithm to be applied on interferograms and a nonlinear algorithm to be applied on unwrapped phase maps. (Shirzaei and Walter, 2011) have used wavelet multi-resolution analysis to distinguish between the effects of orbital errors and other component then they applied a robust regression to estimate the effect of orbital errors as a ramp. (Fialko, 2006) have used more than 50 GPS velocities to remove a linear ramp from a stack of interferograms for the southern San Andreas fault. (Burgmann et al., 2006), working in the San Francisco Bay Area, used a GPS-constrained tectonic model to remove a ramp from the InSAR velocities obtained using persistent scatterer methods (Gourmelen et al., 2010).

The main target of this paper is presenting a new methodology capable of measuring land displacement with geodetic accuracy using only one L-band interferogram and GPS products obtained from online GPS service agencies with application to Tokyo bay area, which has been affected by the 2011 Tohoku Earthquake. All the previous methodologies have adopted one of two main approaches to correct for baseline errors, First, by using orbital models (Zhang et al., 2008) or regression models (Shirzaei and Walter, 2011) which can be applied directly to the interferograms or the unwrapped phase maps. Second, by using GPS stations to model land deformation and correct for the residual artifact in the interferometric deformation map. Unfortunately these two approaches can't be applied to our study area mainly because of the nature of deformations resulted from the 2011 Tohoku Earthquake. The deformation can be subcategorized into two categories, first, the large nonlinear shift of the study area which introduces large interferometric fringes that dominates the entire interferometric deformation map. These fringes have the same characteristics of the baseline errors fringes; this means that the application of any regression model to the interferometric deformation map will contaminate the data by misinter- 
preting part of the deformation pattern as baseline errors which will produce large errors in the final results. Second the local deformations such as land subsidence and liquifactions, these deformations can't be modeled using GPS observation and therefore they will be considered as residual errors and will be removed if we use the second approach for correction.

That's why we are proposing a methodology which can correct for the tropospheric, ionospheric and baseline errors depending on the interferometric phase information only at the GPS station's pixels. The proposed methodology should correct for the tropospheric error using the estimated ZTD obtained from GNSS Earth Observation Network System's (GEONET) observations. Also we present a new method to correct for the ionospheric and baseline errors using the corrected coordinates of GEONET's GPS network, the proposed method not only corrects for the relative errors in InSAR but also corrects for the ambiguity value of the unwrapped phase and produce absolute deformation maps.

The proposed methodology is only valid for areas where dense GPS networks are available, such as Japan and the west coast of the United States. Also, this methodology is most effective in regions where atmospheric and ionospheric signals are dominated by large scale signatures rather than small scale patterns.

(Martinez et al., 2012) have used TerraSAR-X synthetic aperture radar (SAR) images to generate a ground motion map for Tokyo area by means of correlation techniques. Also they used multiple scenes to generate a wide area displacement map for Japan quantitatively compared with GPS data, showing a divergence of about $15 \mathrm{~cm}$. (Ozawa et al., 2011) have shown coseismic and postseismic displacement maps for Japan using GEONET.

In this research we are aiming at measuring land deformations in Tokyo bay area with geodetic accuracy using ALOS-PALSAR single polarized L-band images. Several challenges have been identified; first of all using only two images with one interferogram will result in high noise level in data. Second: the images were acquired using ALOS-PALSAR which has an orbital accuracy of 1 meter (Rosenqvist et al., 2004). This is too coarse to derive displacement vectors in millimeter level; precise orbit information with centimeter level accuracy is required (Bamler \& Hartl 1998). Third: L-band radio waves are very vulnerable regarding ionospheric effect which will introduce additional errors (Meyer, 2010). Fourth: the humidity level in study area introduces tropospheric error to data. Fifth: The nature of deformation in study area, the deformation occurred affected the entire area which eliminates the possibility of identifying a stable region within the image for adequate phase removal or ambiguity determination.

This paper is organized as follows, section 2 describes the study area and GPS data that have been used in this research, section 3 describes the proposed methodology, section 4 presents the application of the proposed methodology to Tokyo bay area using pre-seismic and co-seismic data and finally section 5 is dedicated to conclusions.

\section{Study Area and GPS Data}

This section briefly introduces the study area, the nature of deformation occurred as a result of the 2011 Tohoku Earthquake and the GPS Data which have been used in this research.

\subsection{Study Area}

Japan was struck by an M9.0 megathrust earthquake on March 11, 2011 at 05:46 Universal Time Coordinated (UTC). The whole archipelago has been affected. Disastrous tsunami waves were triggered by the event. Tokyo has suffered from large deformations that affected the entire area. The deformation can be subcategorized into two types; first: the large deformation or shift that affected the entire region and the second is the local deformations that occurred as a result of this large motion such as local subsidence and liquefactions.

\subsection{GPS Data}

Several continuous GPS networks have been established all over the world for monitoring crustal motion. With 30 seconds observation rate several online agencies present additional products such as corrected stations coordinates/velocities, satellite ephemerids, tropospheric delay, ionospheric delay and many others. Stations corrected coordinates and raw GPS observation files (RINEX) have been obtained from GEONET network, operated by the Geospatial Information Authority of Japan (GSI). 


\subsubsection{Corrected Coordinates}

GEONET is Japan's nationwide GPS permanent array established by GSI to monitor crustal deformations and to provide reference stations for land surveying by GPS. It covers the whole area of Japan with more than 1200 continuous permanent GPS stations. The mean distance between the stations is about 25 kilometers (Yamagiwa et al., 2006).

One of GEONET's products is the corrected coordinates of GPS stations nationwide. For every GPS station GEONET presents one file contains the corrected coordinate's value of day-to-day for the entire year. GEONET uses ITRF2005 as reference coordinate frame and GRS-80 as reference ellipsoid.

\subsubsection{RINEX files and Tropospheric Delay estimation}

The tropospheric delay is dependent on temperature, atmospheric pressure and water vapor content. Tropospheric effect can be divided into two components, dry and wet components. The dry component accounts for about $90 \%$ of the tropospheric effect and can be accurately modeled using surface measurements of temperature and pressure.

One of the most popular models to compute the tropospheric delay is Saastamoinen model eq. (1) (Saastamoinen,1973), which is based on the laws associated with ideal gas.

$\Delta e=\frac{0.002277}{\cos z}\left[p+\left(\frac{1255}{T}+0.05\right) e-\tan ^{2} z\right]$

Where the atmospheric pressure $p$ and the partial water vapor pressure $e$ are given in millibars, the temperature $T$ in degrees Kelvin, and $z$ is the zenith angle; the result is given in meters.

However, due to the high variation in the water vapor content, it is very difficult to model the remaining wet component (Janssen et al., 2004). GEONET presents zenithal tropospheric delay (ZTD) estimates for every GPS station nationwide with time interval of three hours. (Janssen et al., 2004) have studied the ZTD time interval effect on tropospheric delay estimation and found that time interval of 5 minutes is the optimum.

For that reason we have used the raw GPS observation files (RINEX) which presents observations every 30 seconds to estimate the ZTD for every GPS station at time interval of 5 minutes. GPS processing and ZTD estimates have been carried out using the online GNSS processing tool presented by (GMV) company (http://www.gmv.com ) (http://magicgnss.gmv.com). (Fourqan, 2010) has proved the accuracy and reliability of this package as a tool for monitoring tropospheric water vapor. For ZTD estimation we have used static precise point positioning (PPP), Saastamoinen model and Niell mapping (Niell, 1996). The estimated coordinates have been verified against the corrected coordinates obtained from GEONET.

\section{Methodology}

We present a methodology using GPS derived products for correcting InSAR unwrapped phase to obtain deformation maps with geodetic accuracy. The methodology is outlined in Fig. 1.

Eq. (2) presents the interferogram phase generated using two single look complex (SLC) images. The interferometric phase contains topography effects $\phi_{\text {Topo }}$ which can be removed using Digital Elevation Model (DEM). In this research we used the Shuttle Radar Topography Mission (SRTM-3) DEM to remove the topography effect presented in interferograms. The interferometric phase also contains deformation effects $\phi_{D e f o r m}$, tropospheric delay effects $\phi_{\text {Tropo }}$, ionospheric delay effects $\phi_{\text {Iono }}$, baseline error effects $\phi_{\text {Baseline }}$ and noise effects $\phi_{\text {Noise }}$.

$\phi=\phi_{\text {Topo }}+\phi_{\text {Deform. }}+\phi_{\text {Tropo }}+\phi_{\text {Iono }}+\phi_{\text {Baseline }}+\phi_{\text {Noise }}$

Tropospheric delay is highly variable and it can affect the entire deformation map with additional local disturbances that depends on many variables such as local altitude, temperature, water vapor and wind. Ionospheric delay is depending - in addition to the radio wave's inverse squared frequency eq. (5) - on the sun activity therefore it can have severe effect and local disturbances in day time and low gradient effect in night time. Baseline 
error can affect the interferometric map with large wave interferometric fringes which can be approximated with first or second degree surface.

After interferogram generation and flattening using DEM, filtered and unwrapped phase maps should be created using suitable techniques. In this research we used Goldstein method for filtering and Minimum Cost Flow (MCF) method for phase unwrapping (Fig. 1, Step A).

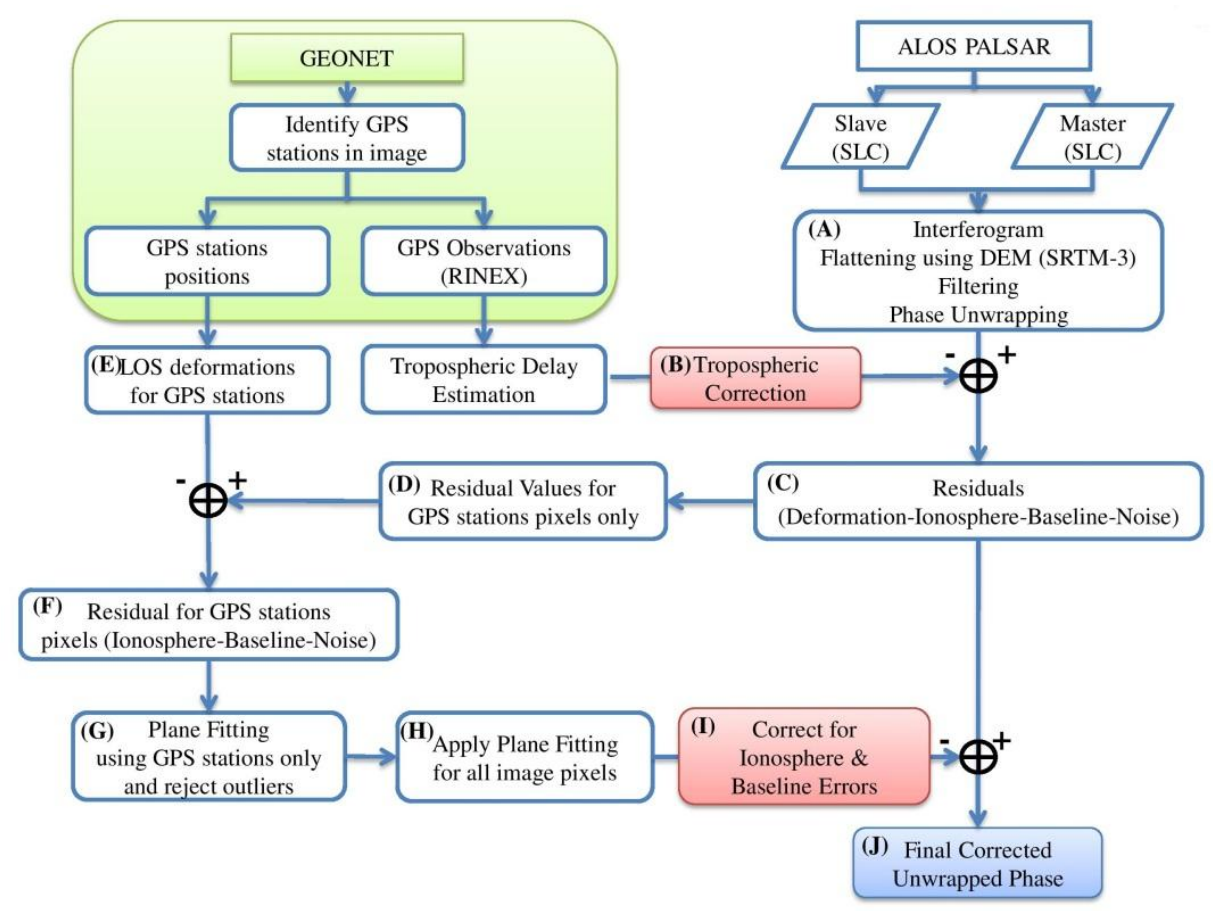

Fig. 1. Methodology block diagram. SLC: Single Look Complex and LOS: Line Of Sight

\subsection{Tropospheric Correction}

The main target of tropospheric correction (Fig. 1, Step B) is to calculate the differential tropospheric delay map for the interferometric SAR unwrapped phase map using ZTD estimated from raw GPS observation files (RINEX) of GEONET.

After identifying the GPS stations located in the PALSAR images we used additional stations around the image boundaries to ensure adequate tropospheric mapping (see Fig. 2.a and Fig. 5.a). GEONET presents the raw GPS observations at 30 seconds interval. First, we collected the raw GPS observation for the entire day of SAR observations of both images then we used PPP processing to estimate ZTD at 5 minutes intervals. Next, we used piecewise cubic Hermite interpolation technique (Carlson \& Fritsch, 1980) to calculate the ZTD value at the exact time of SAR observations, then we calculated the ZTD difference for every GPS station (d ZTD) and multiplied it by a factor of two to account for the 2-way passage of SAR signal through troposphere eq. (3). After that we used these data to generate the differential slant tropospheric delay (d STD) map for the entire scene with spatial resolution equals to the spatial resolution of the unwrapped phase map. We used ordinary kriging method for interpolation and $\left(1 / \cos \theta_{i}\right)$ as mapping function eq. (4) where $\theta_{i}$ is the incident angle of pixel $i$.

$$
\begin{aligned}
& d Z_{\text {GPS station } i}=2 \times\left(Z T D_{\text {GPS station } i}^{\text {Day } 2}-Z T D_{\text {GPS station } i}^{\text {Day } 1}\right) \\
& d S T D=\frac{d Z T D}{\cos \theta_{i}}
\end{aligned}
$$

\subsection{Ionospheric and Baseline Correction}

The ionosphere is a region of the earth's atmosphere extending from an altitude of about $50-1000 \mathrm{~km}$ where solar radiation ionizes atmospheric gases. For the purposes of SAR, it is sufficient to model the ionosphere as a 
thin layer at an effective height of (350-450 km). During its 2-way passage through the dispersive ionosphere, a SAR signal experiences a group delay and phase advance which are inversely proportional to frequency squared eq. (5).

$\tau_{\text {phase }}=-\frac{2 K_{0} S T E C}{f^{2}}[\mathrm{~m}]$

Where $K_{0}=40.28 \mathrm{~m}^{3} \mathrm{~s}^{-2}$ and STEC is the Slant range Total Electron Content (TEC). TEC refers to the zenith value, the density of free electrons contained in a cylinder with unit area passing through the atmosphere in the direction of nadir. STEC is proportional to TEC through $(1 / \cos z)$, where $z$ is the zenith angle. Note that the group delay and phase advance are equal in magnitude but opposite in sign. (Ishimaru et al. , 1999) and (Meyer et al., 2006).

Many methodologies have been developed for estimating and correcting the ionospheric artifact in interferometric SAR. (Freeman, 2004), (Meyer and Nicoll, 2008) and (Rosen et al., 2011) have proposed and used the Faraday rotation methodology to estimate the ionospheric TEC from quad-polarimetric L-band SAR images. (Brcic et al., 2010) and (Brcic et al., 2011) have proposed and successfully applied the split-spectrum method which can be applied on single polarized SAR images.

Our main target in this research is to provide simple yet powerful procedure to measure land deformations with geodetic accuracy taking the advantages of the availability of dense GPS observations. Considering that we have used ALOS-PALSAR single polarized images and the sophisticated procedure of split-spectrum method can be quite complicated to apply, we have decided to assume the ionospheric effect as a linear ramp and combine the ionospheric correction and baseline correction in single step. Another reason to justify our assumption is that we have used only ALOS-PALSAR images in ascending mode. Because of ALOS-PALSAR orbital characteristics the image acquisition time in ascending mode is always at night (22:00, local time) and it's well known that the ionosphere layer activity at night can be considered minimum.

The errors in the satellite's state vectors are not the main error source in interferometry. In fact it is the error in the estimated distance between the two satellite passages which is identified as baseline error. (Massonnet and Feig, 1998) proposed a method to diminish those baseline errors for topography height estimation. However, this method depends on counting the number of fringes along the sides of the interferometric map and correct for the satellite positions accordingly. (Zhang et al., 2008) proposed a bilinear algorithm and a nonlinear algorithm to eliminate the baseline errors. The linear algorithm is done on interferograms, in which the fringes caused by baseline errors is first estimated with Fast Fourier Transform (FFT) in range and azimuth direction and then eliminated. The nonlinear algorithm is performed on unwrapped phase maps, where a quadratic surface function is used to fit the trend of unwrapped phase and then the trend is subtracted. These methods show good results when the deformation is presented locally in interferogram, then any additional fringes can be identified as baseline errors and can be removed.

Unfortunately; this is not the case in our area of study because the 2011 Tohoku Earthquake has affected almost the entire Japanese archipelago, so the deformation fringes is dominant for the entire interferogram and have similar characteristics to the baseline errors fringes, (see Fig. 5.b). Moreover there is no stable area which can be identified for adequate ambiguity determination. That's why we needed a new method to correct for baseline errors in spite of the nature of deformation and adequately determine the ambiguity value.

Our proposed method corrects for ionospheric error, baseline error and ambiguity value using GPS station coordinates. After correcting for tropospheric delay errors the residual unwrapped phase should contain the deformation in addition to ionospheric error, baseline error and noise (Fig. 1, Step C). We identified the locations of GPS stations in the unwrapped phase map (see Fig. 2.a and Fig. 5.a) and extracted the residual values for the GPS stations pixels (Fig. 1, Step D). Using GEONET's corrected coordinates for the GPS stations, the deformation vector for each GPS station was calculated, and by using the incident and azimuth angles for each pixel the line of sight (LOS) unit vector was calculated, then LOS deformation can be calculated by inner product of deformation vector and LOS unit vector (Fig. 1, Step E).

The GPS derived LOS deformation values were considered as the true value. Then we obtained the values corresponding to the each GPS station in the tropospheric corrected unwrapped phase map and by subtracting the GPS derived LOS deformation, the errors in InSAR measurements at GPS stations positions can be obtained 
(Fig. 1, Step F). These errors were assumed to be due to ionospheric and baseline errors and were used to fit a two dimensional surface of the first degree in range and azimuth directions eq. (6).

$\Delta($ Ionosphere \& Baseline $)=a+b \times($ Longitude $)+c \times($ Latitude $)$

First, we used all the GPS stations in primary plane fitting to detect outliers (Fig. 1, Step G). After primary plane fitting residual values from plane equation should be calculated and analyzed statistically. Any GPS station showing residual value higher than three times the standard deviation of the residuals $\left(v_{i}>3 \sigma_{\text {Residuals }}\right)$ should be rejected for two reasons, 1) it may represent unreliable phase information, or 2) it may represent local deformation. By rejecting the outlier values we make sure that we preserved the local deformation represented in InSAR deformation map and any unreliable deformation values can be avoided by choosing a suitable coherence threshold.

After identifying and rejecting outliers, we used the filtered data only to fit a two dimensional surface of the first degree in range and azimuth directions (Fig. 1, Step H). This surface is considered to be the ionospheric and baseline correction (Fig. 1, Step I) in addition it corrects for the ambiguity value, which will produce an absolute deformation map (Fig. 1, Step J) after applying it to the tropospheric corrected unwrapped phase map.

\section{Results}

\subsection{Testing with Pre-seismic data}

The methodology was tested using two images which were acquired before the 2011 Tohoku Earthquake. Using GPS stations it was confirmed that no significant deformations occurred between the two acquisitions.

\subsubsection{Data}

Two SAR images for Tokyo bay area, Japan were obtained from JAXA's ALOS-PALSAR using Fine Beam Single polarization (FBS) mode (see table 1). Daily RINEX observation files and corrected coordinates at the GEONETs' GPS stations were used for tropospheric, ionospheric and baseline errors corrections. 10 GSP stations within image and 26 stations around it with total 36 stations were identified (see Fig. 2.a). Note that; GEONET's GPS products may not be available for some stations at certain observation epochs. That's why the number of GPS stations may be different for each observation depending on the availability of GPS data (see Fig. 2.a and Fig. 5.a).

Interferogram was generated using SARscape software, the effect of topography was removed using SRTM-3 DEM (Fig. 2.b). Goldstein method was used for filtering and Minimum Cost Flow (MCF) method was used for phase unwrapping with coherence threshold equals 0.2 . To reduce the effect of speckles we used multilooking of 3 looks in range and 8 looks in azimuth resulting in pixel size of 22.47 meters in range and 24.45 meters in azimuth. Unwrapped phase map was converted to displacements using eq. (7) (see Fig. 2.c).

$\Delta=-1 * \frac{\phi \times \lambda}{4 \pi}$

Where, $\lambda$ is the wave length and $\phi$ is the unwrapped phase.

Table 1

Details of SAR images used in Pre-seismic analysis

\begin{tabular}{|c|c|c|}
\hline & Master 1 & Slave 1 \\
\hline Observation date \& Time (UTC) & 03 /Apr/ 2010 (13:08:49) & 19 /Aug/ 2010 (13:07:24) \\
\hline Normal Baseline & 593.2 meters \\
\hline Time Baseline & 138 days \\
\hline Satellite/Sensor/Mode/Direction & ALOS / PALSAR / FBS / Ascending \\
\hline Polarization & HH \\
\hline
\end{tabular}



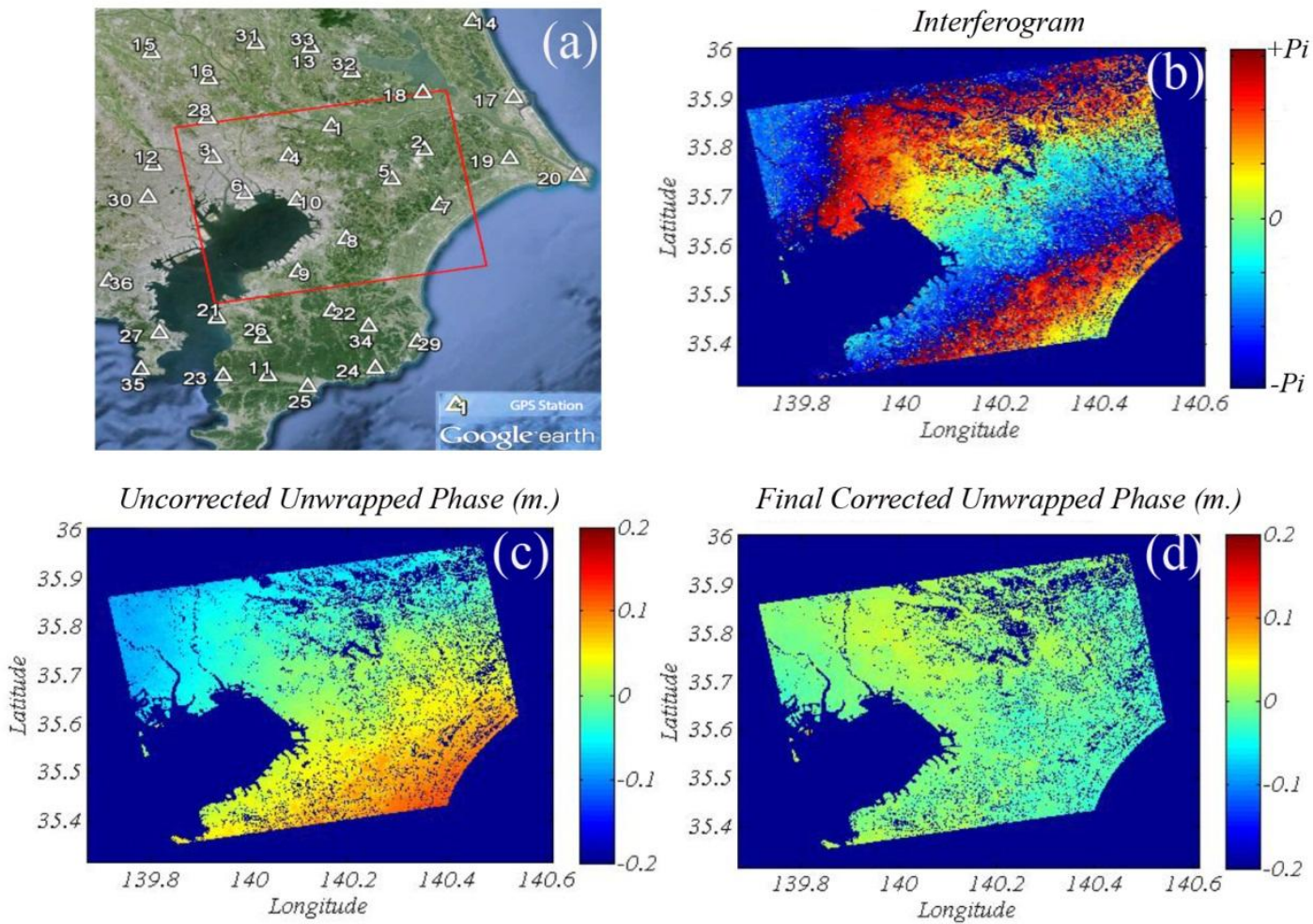

Fig. 2. (a) Study area showing GPS stations, (b) Interferogram, (c) Displacement map in meters and (d) Final corrected displacement map in meters

Table 2

Parameters of planes representing ionosphere and baseline errors in analysis

\begin{tabular}{|c|c|c|c|c|}
\hline Parameters & $\mathrm{a}$ & $\mathrm{b}$ & $\mathrm{c}$ & $\mathrm{R}^{2}$ \\
\hline (1) Pre-seismic & -9.30 & 0.1799 & -0.4639 & 0.9848 \\
\hline (2) Co-seismic & -24.55 & 0.2056 & -0.1155 & 0.9639 \\
\hline
\end{tabular}

\subsubsection{Pre-seismic Results}

ZTD estimates for the 32 GPS stations out of 36 available GPS stations were used for tropospheric delay corrections reserving 4 stations located within the deformation map for accuracy verification. Referring to section 3.1 dSTD estimation was calculated (see Fig. 3.a) and subtracted from the unwrapped phase. Then the values of the unwrapped phase map corresponding to the GPS stations positions after tropospheric correction were identified then we subtracted LOS deformation derived from GPS observations from it. First we made plane fitting using all GPS station, then residual values were calculated and analyzed statistically. All the GPS stations showed residual values $v_{i}$ from the plane equation lower than three times the residuals standard deviation $\left(v_{i}<\right.$ $3 \sigma$ Residuals therefore no GPS stations were rejected. We used only 6 GPS stations for the correction of ionospheric and baseline errors [stations no. 1, 2,3,4,7 and 9] and reserved 4 stations for accuracy verification [stations no. 5, 6, 8, and 10] those are the same stations reserved in troposphere correction stage (see Fig. 2.a). The resulting values were assumed to be due to ionospheric and baseline errors, neglecting noise. First degree plane eq. (6) was fitted to the data (see table 2, (1) Pre-seismic) and considered to be the ionospheric and baseline errors combined (see Fig. 3.b).

After applying tropospheric, ionospheric and baseline error corrections the resulting unwrapped phase is considered the final corrected unwrapped phase (see Fig. 2.d). The contribution percentage of tropospheric error, ionospheric and baseline errors was calculated and presented in Fig. 7 


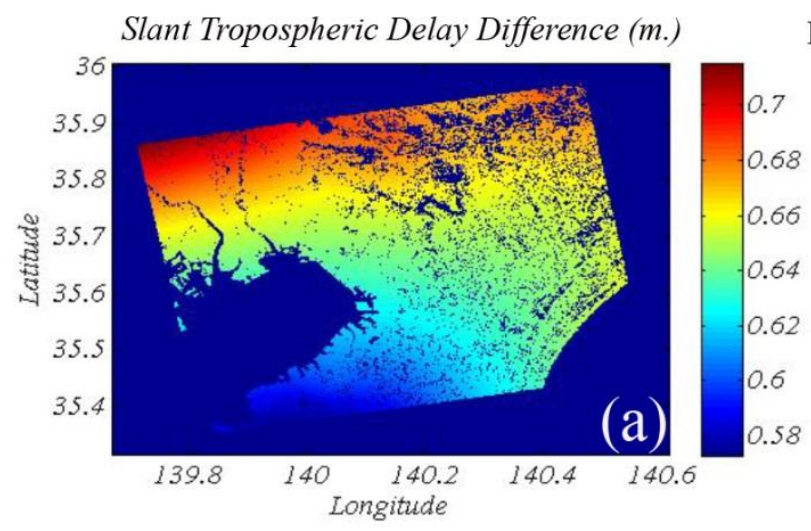

Plane Fitting for Residuals "Ionosphere \& Orbital Errors" (m.)

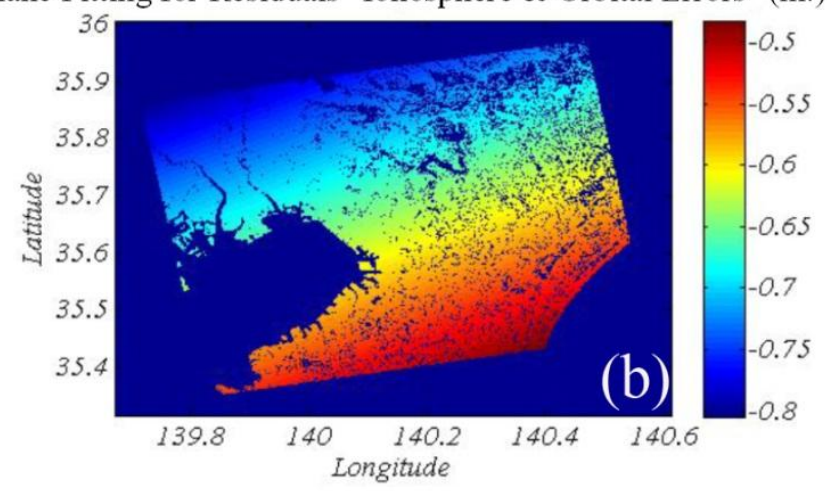

Fig. 3. (a) Slant troposphere delay differenced map in meters multiplied by factor of two, (b) Fitted plane representing ionosphere and baseline errors in unwrapped phase map.

\subsubsection{Accuracy Check}

Accuracy verification has been done by comparing the LOS deformation values at GPS stations positions obtained from the corrected unwrapped phase map and GEONET's corrected coordinates. In addition we calculated the deformation using uncorrected unwrapped phase after removing the ambiguity to present the improvement induced by the proposed methodology.

We found that; the amount of errors is in sub-centimeter level (see Fig. 4) and shows an improvement of $90.0 \%$ in the standard deviation. (See table 3).

To ensure adequate comparison, the ambiguity correction applied to the uncorrected unwrapped phase is considered to be the mean value of overall correction map. The overall correction map is the summation of tropospheric delay correction map (Fig. 3.a), ionospheric and baseline correction map (Fig. 3.b). This method ensures that the same ambiguity value is applied to both uncorrected and corrected unwrapped phase. Any additional shift identified is mainly due to the corrections proposed by our methodology.

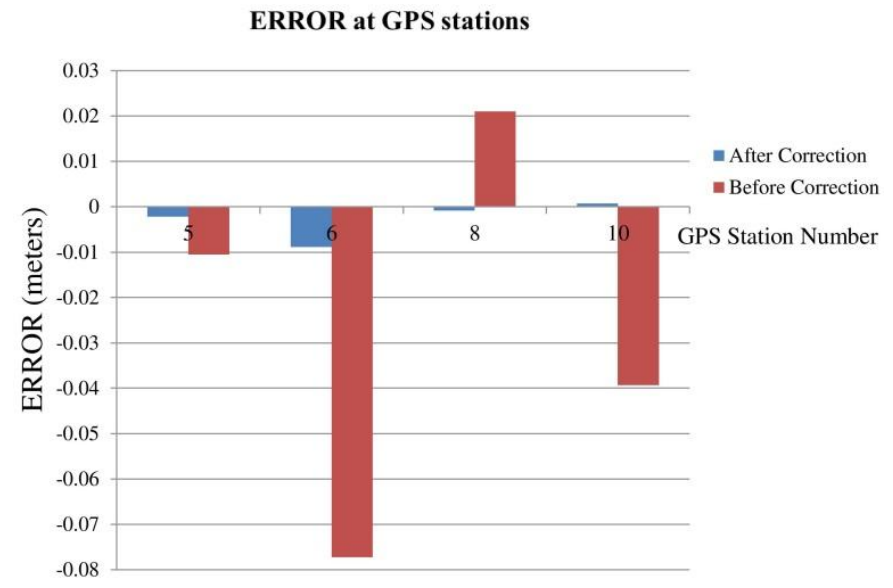

Fig. 4. Pre-seismic unwrapped phase map errors at the GPS stations before and after correction

Table 3

Statistical analysis results for the GPS stations before and after applying the proposed methodology

\begin{tabular}{|c|c|c|c|}
\hline \multirow{2}{*}{} & \multicolumn{3}{|c|}{ Standard Deviation (m.) } \\
\cline { 2 - 4 } & Uncorrected & Corrected & Improvement \\
\hline GPS stations & 0.0418 & 0.0042 & $90.0 \%$ \\
\hline
\end{tabular}




\subsection{Application to Co-seismic data}

\subsubsection{Data}

Two SAR images for Tokyo bay area, Japan obtained from JAXA's ALOS-PALSAR were used (see table 4). Daily RINEX observation files and corrected coordinates at the GEONETs' GPS stations were used for tropospheric, ionospheric and baseline errors corrections, 18 GPS stations within image and 21 stations around it with total 39 stations were identified (see Fig. 5.a).

Interferogram was generated using SARscape software, the effect of topography was removed using SRTM-3 DEM (Fig. 5.b). Goldstein method was used for filtering and Minimum Cost Flow (MCF) method was used for phase unwrapping with coherence threshold equals 0.2 . To reduce the effect of speckles we used multilooking of 3 looks in range and 7 looks in azimuth resulting in pixel size of 22.46 meters in range and 22.88 meters in azimuth. Unwrapped phase map was converted to displacement using eq. (7) (see Fig. 5.c).

Table 4

Details of SAR images used in Co-seismic analysis

\begin{tabular}{|c|c|c|}
\hline & Master 2 & Slave 2 \\
\hline Observation date \& Time (UTC) & $19 / \mathrm{Feb} / 2011$ (13:04:08) & 06 /Apr/ 2011 (13:03:01) \\
\hline Normal Baseline & 396.262 meters \\
\hline Time Baseline & 46 days \\
\hline Satellite/Sensor/Mode/Direction & ALOS / PALSAR / FBS / Ascending \\
\hline Polarization & HH \\
\hline
\end{tabular}
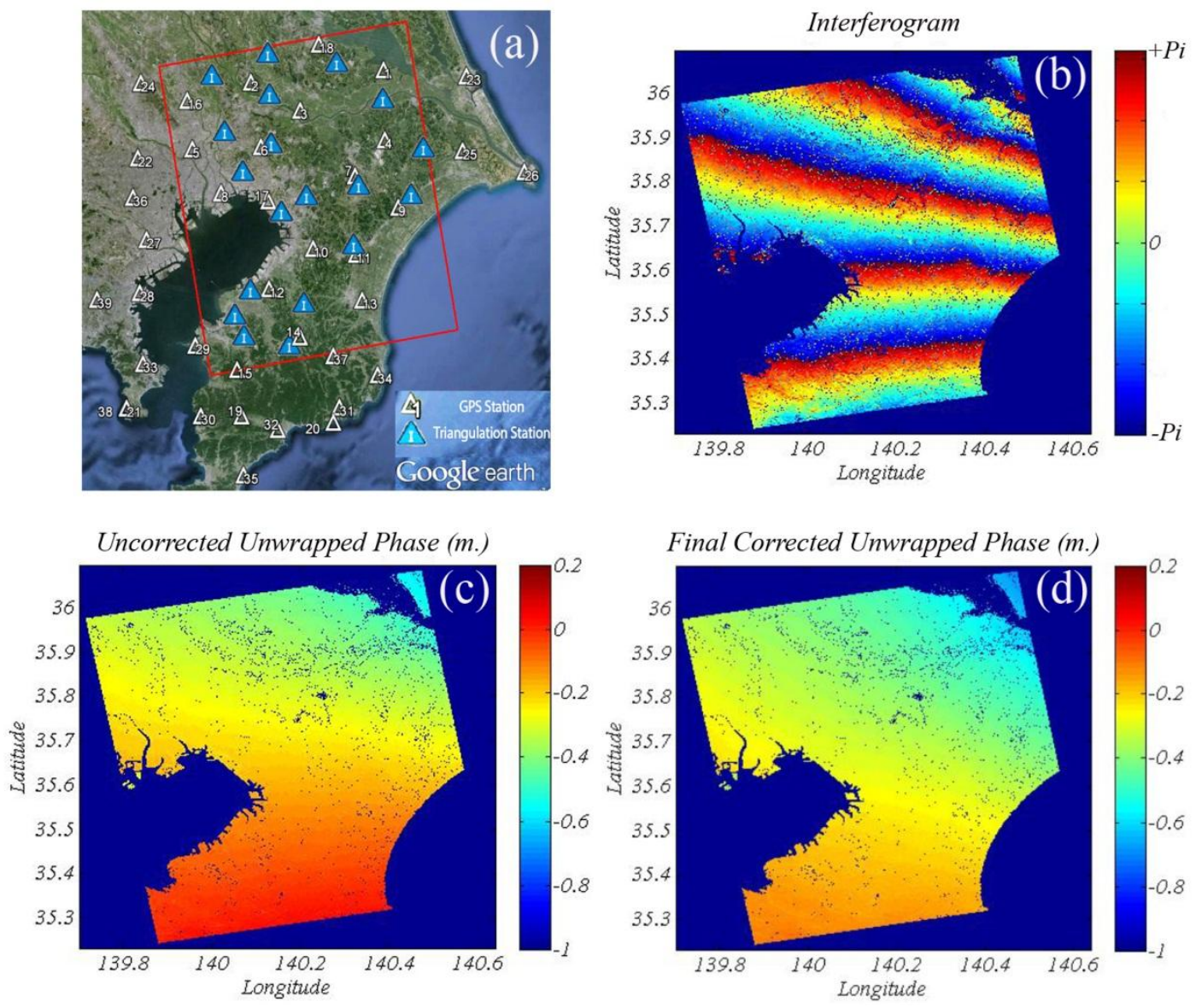

Fig. 5. (a) Study area showing GPS and triangulation stations, (b) Interferogram, (c) Displacement map in meters and (d) Final corrected displacement map in meters. 


\subsubsection{Co-seismic Results}

ZTD estimates for the 31 GPS stations out of 39 available GPS stations were used for tropospheric delay corrections reserving 8 stations located within the deformation map for accuracy verification. Referring to section 3.1 dSTD estimation was calculated (see Fig. 6.a) and subtracted from the unwrapped phase. Then the values of the unwrapped phase map corresponding to the GPS stations positions after tropospheric correction were identified then we subtracted LOS deformation derived from GPS observations from it. First we made plane fitting the residual values using all GPS station the results were calculated and analyzed statistically. GPS stations number 15 and 17 were considered as outlier as their residual values $v_{i}$ from the plane equation is higher than three times the residuals standard deviation $\left(v_{15} \& v_{17}>3 \sigma_{\text {Residuals }}\right)$. Then we used only 8 GPS stations for the correction of ionospheric and baseline errors [stations no. 1,4,6,9,10,14,16 and 18] and reserved 8 stations for accuracy verification [stations no. 2, 3, 5, 7, 8, 11, 12 and 13] those are the same stations reserved in troposphere correction stage (see Fig. 5.a). The resulting values were assumed to be due to ionospheric and baseline errors, neglecting noise. First degree plane eq. (6) was fitted to the data (see table 2, (2) Co-seismic) and considered to be the ionospheric and baseline errors combined (see Fig. 6.b).

After applying tropospheric, ionospheric and baseline error corrections the resulting unwrapped phase is considered the final corrected unwrapped phase (see Fig. 5.d).
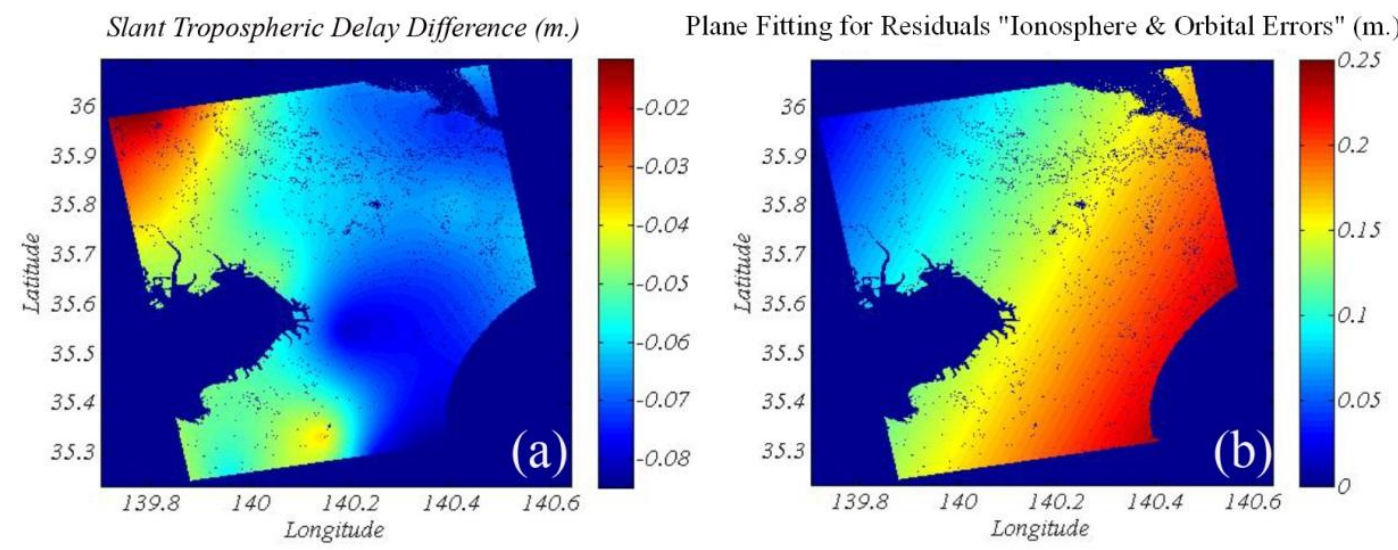

Fig. 6. (a) Slant troposphere delay differenced map in meters multiplied by factor of two, (b) Fitted plane representing ionosphere and baseline errors in unwrapped phase map.

To understand the nature of errors presented in InSAR deformation maps, the percentage of each error source was calculated and presented in Fig. 7. The analysis covered the pre-seismic and co-seismic data. Tropospheric, ionospheric and baseline errors are absolute values. On the other hand the InSAR observed deformation is relative value. That's why it might be tricky to calculate the contribution percentage of each error source to the InSAR deformation map. In this analysis we calculated the relative error for each error source, which means the difference between the maximum and minimum values in the correction maps (Fig. 3.a and Fig. 3.b) for the preseismic data and maps (Fig. 6.a and Fig. 6.b) for the co-seismic data. Then we calculated the contribution percentage of each error source to the total error budget affecting the InSAR deformation maps (see Fig. 7).

It can be seen that the contribution percentage of each error source is variable and changes with time even for the same area. Although the contribution percentage of each error source is depending on several elements such as time, position, sun activity and many others, it can be concluded that in our analysis the ionosphere and baseline errors have the largest contributions in the total error budget affecting the InSAR deformation maps. 


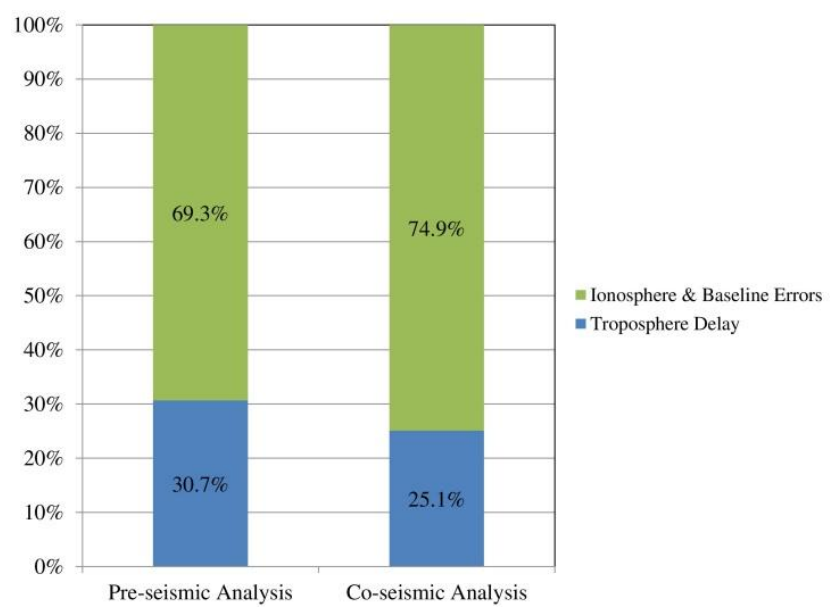

Fig. 7. Contribution percentage of each error source to the total error budget

\subsubsection{Accuracy Check}

\subsubsection{GPS Stations}

Accuracy verification has been done by comparing the LOS deformation values at GPS stations positions obtained from the corrected unwrapped phase map and GEONET's corrected coordinates. In addition we calculated the deformation using uncorrected unwrapped phase after removing the ambiguity to present the improvement induced by the proposed methodology.

We found that; the amount of errors is in sub-centimeter level (see Fig. 8 and Fig. 9.a) and shows an improvement of $87.6 \%$ in the standard deviation (see table 5, a).

\section{ERROR at GPS stations}

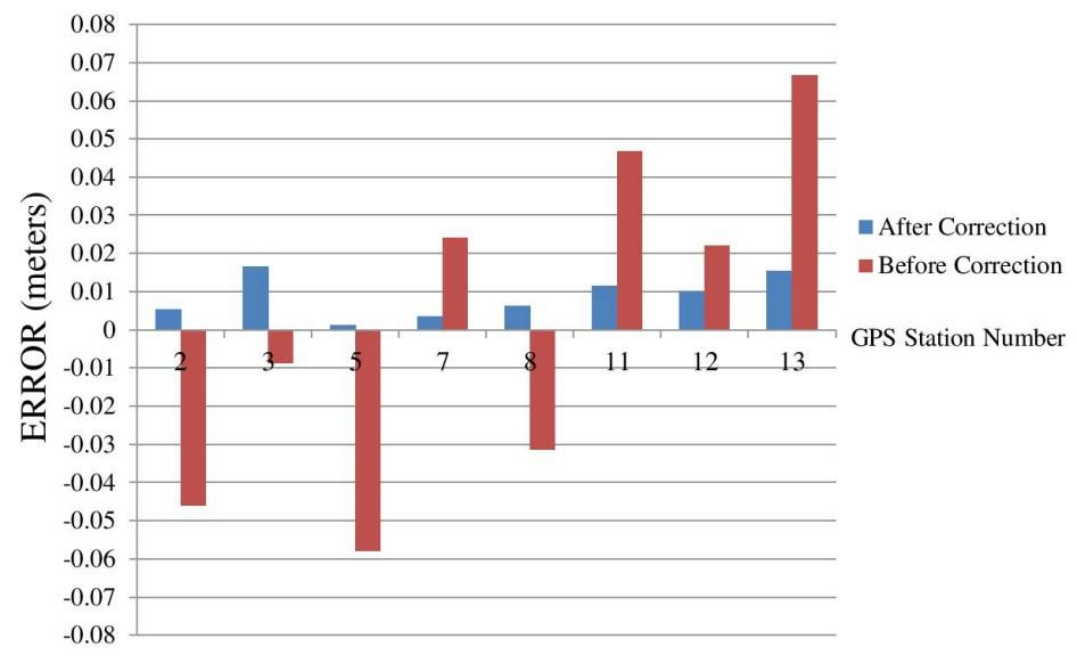

Fig. 8 Co-seismic unwrapped phase map errors at the GPS stations before and after correction

\subsubsection{Triangulation Stations}

Additional accuracy verification has been done using the coordinates of triangulation network of first and second degrees observed by GSI (see Fig. 5.a).

The main observation for geodetic triangulation networks is the horizontal angles. That's why triangulation stations' coordinates have very high accuracy in the horizontal direction and very poor accuracy in the vertical direction. For that reason we used only the horizontal coordinates of the triangulation stations in accuracy verification analysis. 21 stations have been used for verification by comparing its horizontal deformation with the horizontal deformation obtained by final corrected unwrapped phase. 
For the triangulation network stations, the deformation component in LOS direction was calculated first. Then deformations in the horizontal direction can be obtained using eq. (8).

The corresponding InSAR horizontal deformations were calculated using the final corrected LOS deformation map and incident $\left(\theta_{i}\right)$ angle for each station eq. (8). In addition we calculated the deformation using uncorrected unwrapped phase after removing the ambiguity. (See Fig. 9.b).

$\Delta \mathrm{H}=$ Deformation $_{\mathrm{LOS}} \times \sin \left(\theta_{i}\right)$

The difference between the values obtained from the corrected unwrapped phase and triangulation stations were calculated and analyzed statistically. Triangulation stations no. 11, 12 and 20 were considered as an outlier as their residual values $v$ are higher than three times the residuals standard deviation $v_{11}, v_{12} \& v_{20}>$ $3 \sigma_{\text {Residuals }}$. Triangulation station no. 19 was neglected because it has no corresponding value in unwrapped phase map (NaN value)
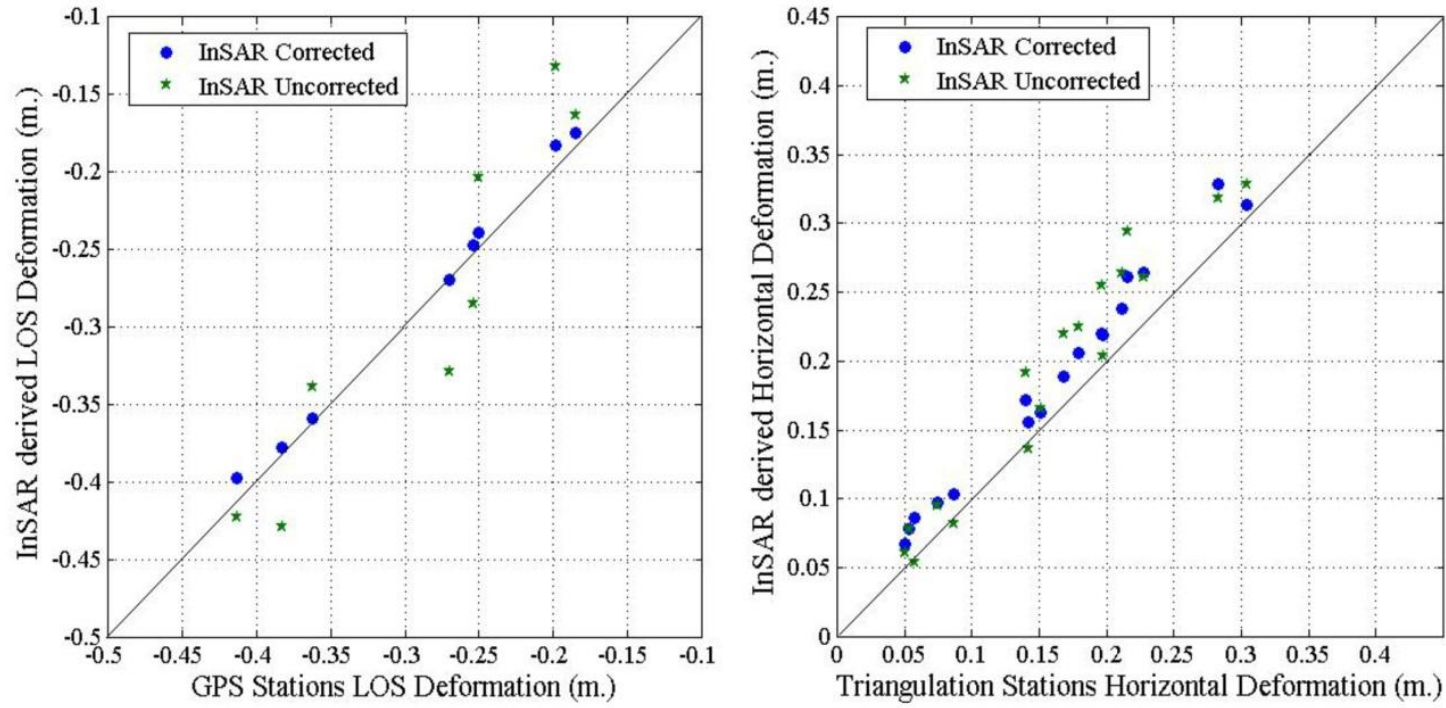

Fig. 9. (a) LOS deformations obtained by GPS and unwrapped phase map before and after applying the methodology, positive values means motion towards the satellite. (b) Horizontal deformations obtained by triangulation stations and unwrapped phase map before and after applying the methodology, positive values means motion towards East or North.

\section{Table 5}

Statistical analysis results for the GPS and Triangulation stations before and after applying the proposed methodology.

\begin{tabular}{|ll|c|c|c|}
\hline \multirow{2}{*}{} & \multicolumn{3}{|c|}{ Standard Deviation (m.) } \\
\cline { 3 - 5 } a. & GPS stations & 0.0450 & 0.0056 & $87.6 \%$ \\
\hline b. & Triangulation Stations & 0.0251 & 0.0105 & $58.2 \%$ \\
\hline
\end{tabular}

(Table 5, b) shows a statistical analysis of the error in InSAR obtained horizontal deformation before and after applying our proposed methodology considering that the true deformation value is presented by triangulation stations coordinates.

If we studied (Fig. 9.b) carefully, we will find that the deformation values are shifted towards the InSAR observed deformation. That is understandable because geodetic triangulation network didn't detect nearly any deformation in the vertical direction because it's main purpose is to measure deformations in horizontal direction only, meanwhile InSAR can detect the vertical deformation. For more clarification, if we considered a geodetic triangulation station that suffered only from vertical deformation downwards equal to $(-\Delta h)$ the horizontal observation of the triangulation station will not detect any motion. On the other hand InSAR will detect change in range in LOS direction will equal to $\left(\Delta L O S=-\Delta h \times \cos \theta_{i}\right)$, where $\theta_{i}$ is the InSAR incident angle at the triangulation station position. That deformation will be projected to the absolute horizontal deformation detected by 
InSAR according to eq. (8). Which will make the horizontal deformation detected by InSAR larger that the horizontal deformation detected by triangulation stations. And that case is presented in (Fig. 9.b).

For confirmation purpose we have interpolated the vertical deformations at triangulation stations using GPS stations' observations and ordinary kriging. Then we calculated the horizontal deformation of triangulation stations and compared it against the horizontal deformation obtained from the corrected InSAR deformation map. (Fig. 10) shows that the shift has been corrected, indicating that our interpretation of (Fig. 9.b) is correct. The standard deviation of the errors in (Fig. 10) has increased to 14.9 millimeters because of the inherited errors in the interpolated vertical deformations.

This analysis shows lower accuracy than the GPS accuracy check. That is understandable because the triangulation stations coordinates were not observed at the exact time of SAR observation, actually it was observed several years before the first SAR image and by nearly two months after the second SAR image. That's why we concluded that although this check proves to a very good extent the reliability of our proposed methodology yet we claim that the expected accuracy of this methodology is much better than what presented in this analysis.

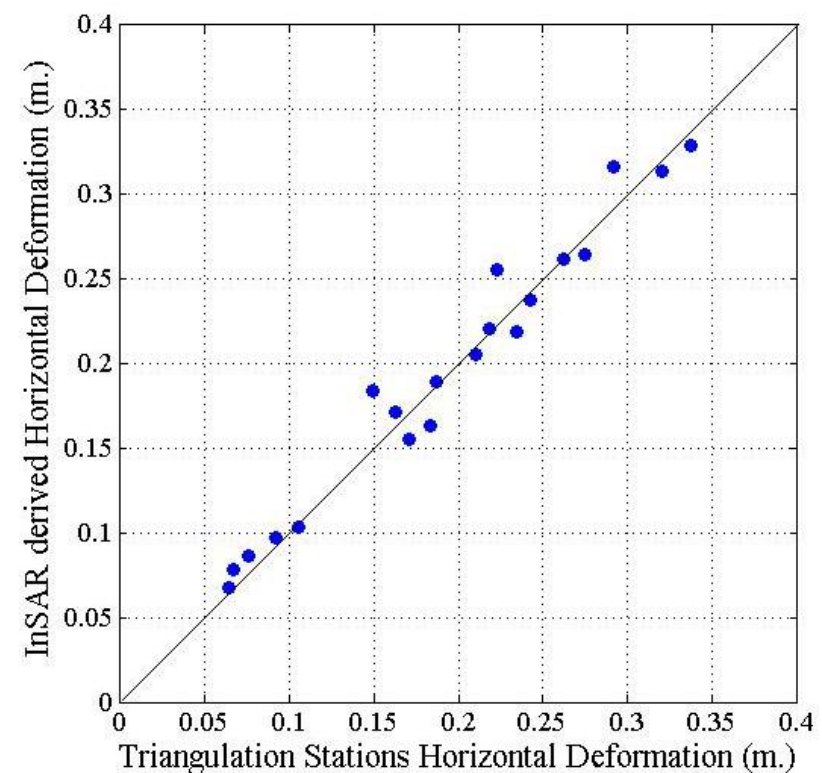

Fig. 10. Horizontal deformations obtained by triangulation stations (with interpolated vertical deformation component from GPS stations observations) and corrected InSAR deformation map, positive values means motion towards East or North.

\section{Conclusions}

A new methodology has been presented for observing land deformation with geodetic accuracy. The presented methodology merges GPS data products with single L-band interferogram and presents absolute deformation measurements with sub-centimeter accuracy.

The methodology first corrects for the tropospheric delay using ZTD estimated from GEONET's observations. Then it uses the corrected coordinates of GEONET's GPS stations to correct for ionosphere, baseline errors and ambiguity value.

The methodology have been tested in Tokyo bay area before the 2011 Tohoku earthquake and the final results were compared against GPS stations observations, the standard deviation of the residuals was 4.2 millimeters. Then this methodology have been applied in Tokyo bay area to determine the deformations resulting from the 2011 Tohoku earthquake, the final results were compared against GPS stations and triangulation stations observations and the standard deviation of the residuals was 5.6 and 10.5 millimeters, respectively. The lower accuracy of the triangulation station analysis was due to the fact that triangulation stations coordinates were not observed at the exact time of SAR observations. 


\section{Acknowledgment}

We are grateful to the Geospatial Information Authority of Japan (GSI) for the Triangulation stations displacements, GPS displacements and raw GPS observation data used in this study.

\section{References:}

Bamler, R., \& Hartl, P., 1998. Synthetic Aperture Radar Interferometry. Inverse Problems, R1-R54.

Brcic, R., Parizzi, A., Eineder, M., Bamler, R., \& Meyer, F., 2010. Estimation and Compensation of Ionospheric Delay for SAR Interferometry. IGARSS (pp. 2908-2911). IEEE.

Brcic, R., Parizzi, A., Eineder, M., Bamler, R., \& Meyer, F., 2011. IONOSPHERIC EFFECTS IN SAR INTERFEROMETRY: AN ANALYSIS AND COMPARISON OF METHODS FOR THEIR ESTIMATION. Geoscince and Remote Sensing Symposium (IGARSS) (pp. 1497-1500). Vancouver, Canada: IEEE International.

Buckley, S. M., Rosen, P., Hensley, S., \& Tapley, B., 2003. Land subsidence in Houston, Texas, measured by radar interferometry. JOURNAL OF GEOPHYSICAL RESEARCH, VOL. 108, 8-1:8-12.

Burgmann, R., Hilley, G., Ferretti, A., \& Novali, F., 2006. Resolving vertical tectonics in the San Francisco Bay Area from permanent scatterer InSAR and GPS analysis. Geology, VOL.34, 221-224.

Carlson, R. E., \& Fritsch, F. N., 1980. Monotone Piecewise Bicubic Interpolation. SIAM J. Numerical Analysis, 238-246.

Fialko, Y., 2006. Interseismic strain accumulation and the earthquake potential on the southern San Andreas fault system. Nature, VOL.441, 968-971.

Freeman, A., 2004. Calibration of Linearly Polarized Polarimetric SAR Data Subject to Faraday Rotation. IEEE TRANSACTIONS ON GEOSCIENCE AND REMOTE SENSING, VOL.42, 1617-1624.

Furqan, A., 2010. Evaluation of GNSS as a Tool for Monitoring Tropospheric Water Vapour. Master of Science Thesis in the Master Degree Programme, Radio and Space Science, CHALMERS UNIVERSITY OF TECHNOLOGY, Department of Earth and Space Sciences,Group of Space Geodesy and Geodynamics, Goteborg, Sweden.

Goldstein, R. M., Engelhardt, H., Kamb, B., \& Frolich, R. M., 1993. Satellite Radar Interferometry for Monitoring Ice Sheet Motion: Application to an Antarctic Ice Stream. SCIENCE, VOL.262, 1525-1530.

Gourmelen, N., Amelung, F., \& Lanari, R., 2010. Interferometric synthetic aperture radar-GPS integration: Interseismic strain accumulation across the Hunter Mountain fault in the eastern California shear zone. JOURNAL OF GEOPHYSICAL RESEARCH, VOL. 111, B09408.

Ishimam, A., Kuga, Y., \& Liu , J., 1999. Ionospheric effects on synthetic aperture radar at $100 \mathrm{MHz}$ to $2 \mathrm{GHz}$. Radio Science, 257-268.

Janssen, V., Ge, L., \& Rizos, C., 2004. Tropospheric Corrections to SAR Interferometry from GPS Observations. GPS Solutions, 140-151.

Li, Z. W., Ding, X. L., \& Liu, G. X., 2004. Modelling atmospheric effects on InSAR with meteorological and continuous GPS observations: algorithms and some test results. Journal of Atmospheric and SolarTerrestrial Physics, VOL. 66(11), 907-917.

Li, Z., Fielding, E., Cross, P., \& Muller, J.-P. , 2006. Interferometric synthetic aperture radar atmospheric correc-tion:GPS topography-dependent turbulence model. JOURNAL OF GEOPHYSICAL RESEARCH, VOL. 110, B03410.

Martinez, N. Y., Eineder, M., Cong, X. Y., \& Minet, C., 2012. Ground Displacement Measurement by TerraSAR-X Image Correlation: The 2011 Tohoku-Oki Earthquake. IEEE GEOSCIENCE AND REMOTE SENSING LETTERS, 539-543.

Massonnet, D., \& Feigl, K. L., 1998. Radar Interferometry and its Application to Changes in the Earth's Surface. REVIEWS OF GEOPHYSICS, VOL. 36, 441-500.

Massonnet, D., Feigl, K., Marc, R., \& Adragna, F., 1994. Radar interferometric mapping of deformation in the year after the Landers earthquake. Nature, VOL. 369, 227-230. 
Meyer, F., 2010. A Review of Ionospheric Effects in Low-Frequency SAR - Signals, Correction Methods, and Performance Requirements. Geoscinece and Remote Sensing Symposium (IGARSS) (pp. 29-32). Honolulu, Hawaii, USA: IEEE International.

Meyer, F. J., \& Nicoll, J. B., 2008. Prediction, Detection, and Correction of Faraday Rotation in FullPolarimetric L-Band SAR Data. IEEE TRANSACTIONS ON GEOSCIENCE AND REMOTE SENSING, 3076-3086.

Meyer, F., Bamler, R., Jakowski, N., \& Fritz, T., 2006. The Potential of Low-Frequency SAR Systems for Mapping Ionospheric TEC Distributions. IEEE GEOSCIENCE AND REMOTE SENSING LETTERS, 560-564.

Niell, A. E., 1996. Global mapping functions for the atmosphere delay at radio wavelengths. JOURNAL OF GEOPHYSICAL RESEARCH, VOL. 101, 3227-3246.

Onn, F., \& Zebker, H. A., 2006. Correction for interferometric synthetic aperture radar atmospheric phase artifacts using time series of zenith wet delay observations from a GPS network. JOURNAL OF GEOPHYSICAL RESEARCH, VOL. 111, B09102.

Ozawa, S., Nishimura, T., Suito, H., Kobayashi, T., Tobita, M., \& Imakiire, T., 2011. Coseismic and postseismic slip of the 2011 magnitude-9 Tohoku-Oki earthquake. Nature, 373-377.

Rosen, P., Lavalle, M., Pi, X., Buckley, S., Szeliga, W., Zebker, H., et al., 2011. TECHNIQUES AND TOOLS FOR ESTIMATING IONOSPHERIC EFFECTS IN INTERFEROMETRIC AND POLARIMETRIC SAR DATA. IGARSS (pp. 1501-1504). Vancouver, Canada: IEEE.

Rosenqvist, A., Shimada, M., \& Watanabe, M., 2004. ALOS PALSAR:Technical outline and mission concepts. 4th International Symposium on Retrieval of Bio- and Geophysical Parameters from SAR Data for Land Applications. Innsbruck, Austria.

Saastamoinen, J., 1973. Contributions to the theory of atmospheric refraction. Bull Geodesique 107, 13-34.

Shirzaei, M., \& Walter, T. R., 2011. Estimating the Effect of Satellite Orbital Error Using Wavelet-Based Robust Regression Applied to InSAR Deformation Data. IEEE TRANSACTIONS ON GEOSCIENCE AND REMOTE SENSING, VOL. 49(11), 4600-4605.

Song, X., Li, D., \& Liao, M., 2008. REPRODUCTION OF INSAR ATMOSPHERIC SIGNAL USING GPS DATA BASED ON TOPOGRAPHY-DEPENDENT AND TURBULENT MIXING MODEL. 2008 Dragon Symposium, Dragon 1 Programme, Final Results 2004 (ESA SP-655, April 2008). Beijing, P.R. China: ESA.

Xu, C., Wang, H., Ge, L., Yonezawa, C., \& Cheng, P., 2006. InSAR tropospheric delay mitigation by GPS observations:A case study in Tokyo area. Journal of Atmospheric and Solar-Terrestrial Physics, VOL. 68(6), 629-638.

Yamagiwa, A., Hatanaka, Y., Yutsudo, T., \& Miyahara, B., 2006. Real-time capability of GEONET system and its application to crust monitoring. Bulletin of the Geogrephical Survey Institute, 27-33.

Zhang, S., Li, T., Liu, J., \& Xia, Y., 2008. Research on the Linear and Nonlinear Methods of Correcting Baseline Errors on SAR Interferograms. The International Archives of the Photogrammetry, Remote Sensing and Spatial Information Sciences, 161-164. 